\title{
Influence of beta-blockers on endothelial function: A meta-analysis of randomized controlled trials
}

\author{
Michał Peller, Krzysztof Ozierański, Paweł Balsam, \\ Marcin Grabowski, Krzysztof J. Filipiak, Grzegorz Opolski \\ $1^{\text {st }}$ Department of Cardiology, Medical University of Warsaw, Warsaw, Poland
}

\begin{abstract}
Background: Endothelial dysfunction (ED) frequently precedes cardiovascular diseases (CVD) and is a well-established risk factor of major adverse cardiac events. Beta-blockers are the fundamental drugs used in CVD treatment.

Methods: A systematic literature search for randomized controlled trials investigating influence of beta-blockers on endothelial function assessed by flow-mediated dilation (FMD) was performed in the PubMed and Cochrane Databases.

Results: Sixteen full-text studies involving a total of 1,273 patients were included in the final analysis. The mean age of participating patients ranged from 44.9 to 63.2 years, the follow-up duration from 1 to 12 months. The comparison of FMD change between the beta-blockers and placebo groups showed a statistically significant effect of beta-blockers on endothelial function (mean difference $[M D] 0.83 ; 95 \%$ confidence interval $[C I]$ 0.11-1.55; $p=0.02$ ). Third generation beta-blockers improved FMD in a statistically significant manner compared with second generation beta-blockers (MD 1.65; 95\% CI 0.17-3.11; $p=0.03$ ). Beta-blockers gave an FMD change similar to that obtained with angiotensin receptor blockers $(A R B)$, calcium channel blockers (CCB) or hydrochlorothiazide. The FMD value in the beta-blocker group was significantly lower compared with the group treated with angiotensin converting enzyme inhibitors (ACEI) (MD-0.79; 95\% CI-1.37-(-0.21); $p=0.008)$ and higher than in the ivabradine group $(1.6 \pm 3.61$ vs $-0.3 \pm 1.66 ; p=0.02)$.
\end{abstract}

Conclusions: Beta-blockers improve the endothelial function compared with placebo. Moreover, third generation beta-blockers improve FMD values significantly better than the second generation ones. Beta-blockers had similar effect on endothelial function as did ARB, CCB or diuretics. However, the beneficial effect of beta-blockers was lower when confronted with ACEI. (Cardiol J 2015; 22, 6: 708-716)

Key words: flow mediated dilation, beta-blocker, endothelial dysfunction, meta-analysis, arterial hypertension

\section{Introduction}

Endothelium is the innermost layer of blood vessels responsible for regulation of blood flow, inflammation response and hemostasis. Endothelial dysfunction (ED) frequently precedes cardiovascular diseases (CVD) and is a well-established risk factor of major adverse cardiac events [1]. Nowadays, one can assess endothelial function using invasive or non-invasive procedures. One of

Address for correspondence: Paweł Balsam, MD, PhD, $1^{\text {st }}$ Department of Cardiology, Medical University of Warsaw, ul. Banacha 1a, 02-097 Warszawa, Poland, e-mail: pawel@balsam.com.pl 
the most popular methods of endothelial function assessment is still flow-mediated dilatation (FMD). In this method, the brachial artery diameter is measured before and after endothelial-dependent vasodilatation provoked by temporary ischemia.

Beta-blockers were introduced to the therapy in the 1960s. Their effectiveness in the therapy of hypertension, coronary heart disease and heart failure is well documented [2,3]. Of the two types of beta-adrenergic receptors, one (beta1) is mainly found in the heart. The effects of beta1-receptor stimulation include change of chronotropy (increased heart rate), inotropy (increased force of heart contraction), lusitropy (increased myocardial relaxation) and dromotropy (increased conduction speed). Modulation of any of these attributes by beta-blockers can be a goal of a CVD therapy.

Three generations of beta-blockers are available: non-selective, selective and beta-blockers with additional vasodilation properties. Among the $3^{\text {rd }}$ generation beta-blockers, are those with a nitric oxide-dependent vasodilatory activity, which can be crucial for endothelial function improvement [4].

The reversible character of ED is well known [5]. In this paper, we attempted to assess the effectiveness of beta-blockers in improving endothelial function. Additionally, a comparison between particular generations of beta-blockers and other popular groups of cardiovascular treatment agents was performed according to their effects on endothelial function based on published randomized controlled trials.

\section{Materials and methods}

\section{Study selection}

Two co-authors independently searched the PubMed Database and the Cochrane Central Register of Controlled Trials (CENTRAL) for randomized controlled trials published before March 2014. To find suitable studies we used the following search formula: [(endothelial function) OR (flow mediated dilation)] AND [(beta-blockers) OR (beta andrenolytics) OR acebutolol OR atenolol OR betaxolol OR bisoprolol OR carvedilol OR labetalol OR metoprolol OR nadolol OR nebivolol OR pindolol OR propranolol OR sotalol)]. After selection of full text articles, selected references in particular studies were also analyzed.

\section{Inclusion criteria}

We included only studies/reports fulfilling simultaneously all of the following criteria: 1) randomized, 2) controlled with placebo or other active treatment agents, 3) parallel or cross-over trials with 4) double or single blinding or endpoint blinding; 5) in at least one of the arms of the study, beta-blocker administration had to be newly started; 6) ultrasound FMD measures were performed at least twice: before and after treatment; 7) diagnosis of CVD and/or diabetes mellitus in the study group was essential for trial inclusion; 8) published in English between January 1992 and March 2014. Characteristics of the trials included in the present meta-analysis are shown in Table 1. The quality of the trials was assessed by the Effective Public Health Practice Project Quality Assessment Tool [6]. Quality of the majority of the studies was assessed as strong.

\section{Data extraction}

Titles, abstracts and full texts indicated by the search engines used were independently reviewed by two co-authors. After selection of full texts, consensus between these reviewers was necessary for study inclusion. In case of any discrepancies, decision of a third reviewer was binding. For each study, we collected the following information: first author, number of patients in each arm of the study, their age and sex distribution, diagnosed diseases, treatment agents with doses, time of follow-up and FMD values before and after treatment. In case of two or more FMD measurements during the treatment period, the last one was considered. To obtain missing data, e-mails to corresponding authors were sent. When no information about exact mean FMD values was provided, we performed an evaluation based on figures or graphs.

\section{Statistical analysis}

The meta-analysis was performed based on generic inverse variance according to each study. For each study, mean changes of FMD values and standard deviations were calculated. To calculate standard deviation within repeated measurements, we based on the difference between mean values of FMD and the p-value for a paired t-test. P-values were converted to t-values using SAS 9.2. software. When no exact p-values could be obtained, bounded values were assumed. For non-statistically significant differences between baseline and final FMD measurements, the correlation coefficient between the measurements was presumed to be 0.5 . When an interquartile range was reported, we converted it to standard deviation based on Cochrane recommendations [7]. For studies with more than one arm with the same group of drugs, we combined groups and assumed a single pair-wise comparison. 


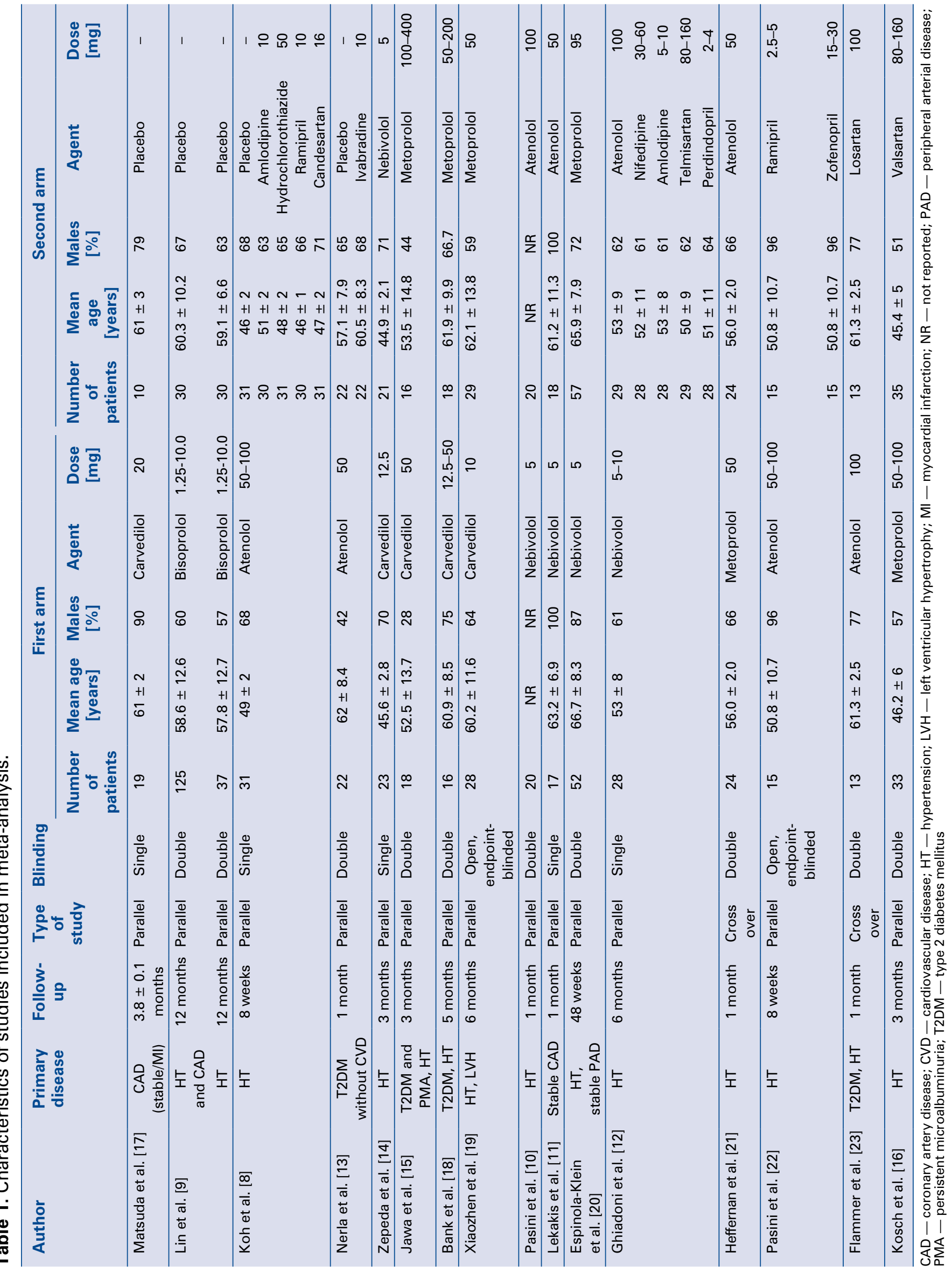




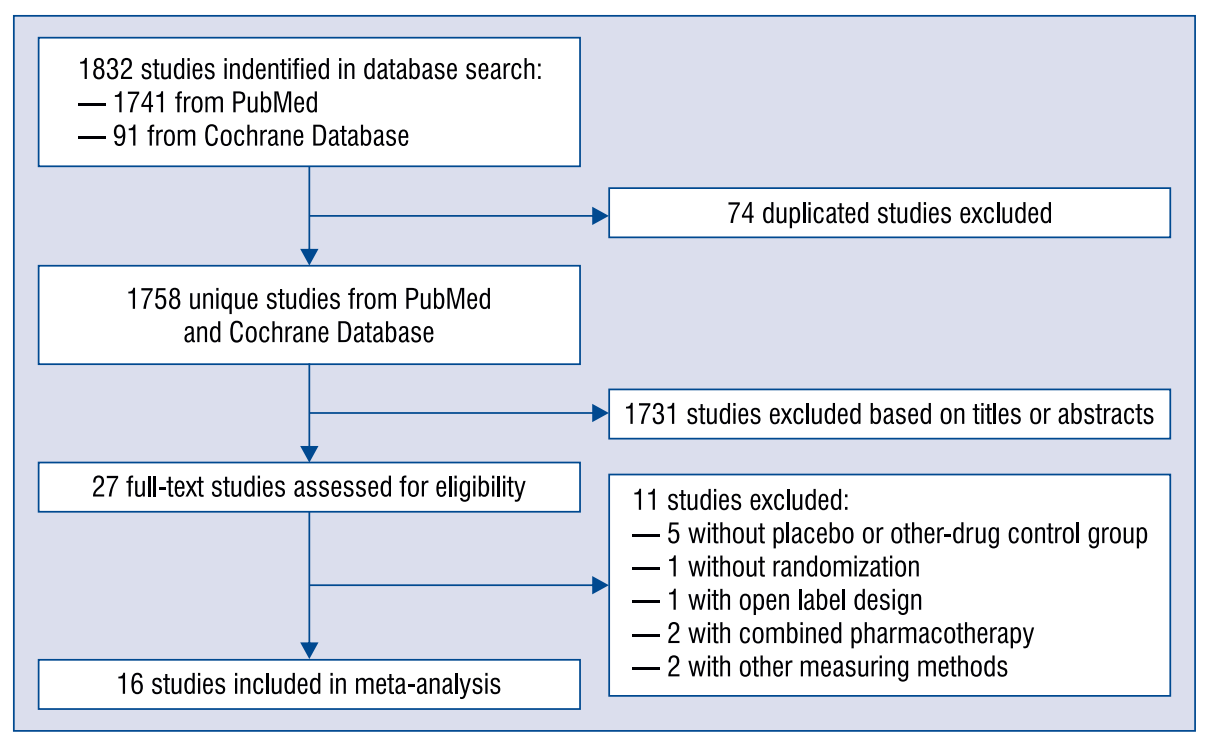

Figure 1. Consecutive steps of study selection.

Heterogeneity $\left(\mathrm{I}^{2}\right)$ between studies was assessed with $\chi^{2}$ test for heterogeneity [7]. For heterogeneity level $>50 \%$ random-effects model was used, otherwise, fixed-effects model was presumed. Statistical significance of pooled treatment effect was considered for $\mathrm{p}$-values $<0.05$. The general part of the meta-analysis was calculated using Review Manager 5.2 (The Cochrane Collaboration).

\section{Results}

\section{Criteria for study inclusion}

Based on the search formula, we found 1,741 studies in the PubMed Database and 91 studies in the Cochrane Database. Altogether, the two co-authors selected independently overall 27 fulltext studies for further analysis. After a careful assessment of the trials, 11 were excluded from the meta-analysis: 5 without placebo or other-drug control group, 1 without randomization, 1 with open label design, 2 with combined pharmacotherapy, 1 with endothelial function assessed using Peripheral Arterial Tonometry and 1 with FMD values adjusted for brachial artery diameter. A total of 1,273 patients from 16 studies were finally included into the meta-analysis. Their mean ages in those groups ranged from 44.9 to 63.2 years. The follow-up duration varied from 1 to 12 months, with 13 of the 16 studies (81.3\%) focused on patients with diagnosed hypertension. The flowchart in Figure 1 illustrates the consecutive steps of study selection.

\section{FMD measurements}

The duration of ischemia and cuff position during FMD measurement was reported in 15 of the 16 studies (except for Koh et al. [8]). In 14 out of 15 studies, the ischemia lasted for $5 \mathrm{~min}$, in 1 study it was 4.5 min [9]. In 12 studies, the cuff was placed on the forearm and in 3 studies measurements was performed with cuff placed on the arm [10-12]. Six studies reported the number of observers who assessed FMD [9, 10, 13-16]. In 5 of those studies, only 1 person was responsible for the FMD measurements. The FMD values before and after treatment and their changes are gathered in Table 2 .

\section{Beta-blockers vs. placebo}

Four out of 16 studies compared the FMD change between the beta-blockers and placebo groups comprising a total of 360 patients [8, 9, 13, 17]. Period between baseline and final FMD measurements ranged from 1 to 12 months. The 2 subgroups of patients analyzed by Lin et al. [9]: the one with coronary artery disease and hypertension and the other with hypertension alone, were combined into one group.

The main conclusion for this analysis is that there was a statistically significant effect of betablockers treatment on the endothelial function compared to placebo, with mean difference (MD) of 0.83 ; 95\% confidence interval (CI) $0.11-1.55$; $\mathrm{p}=0.02$. The heterogeneity of those studies was 


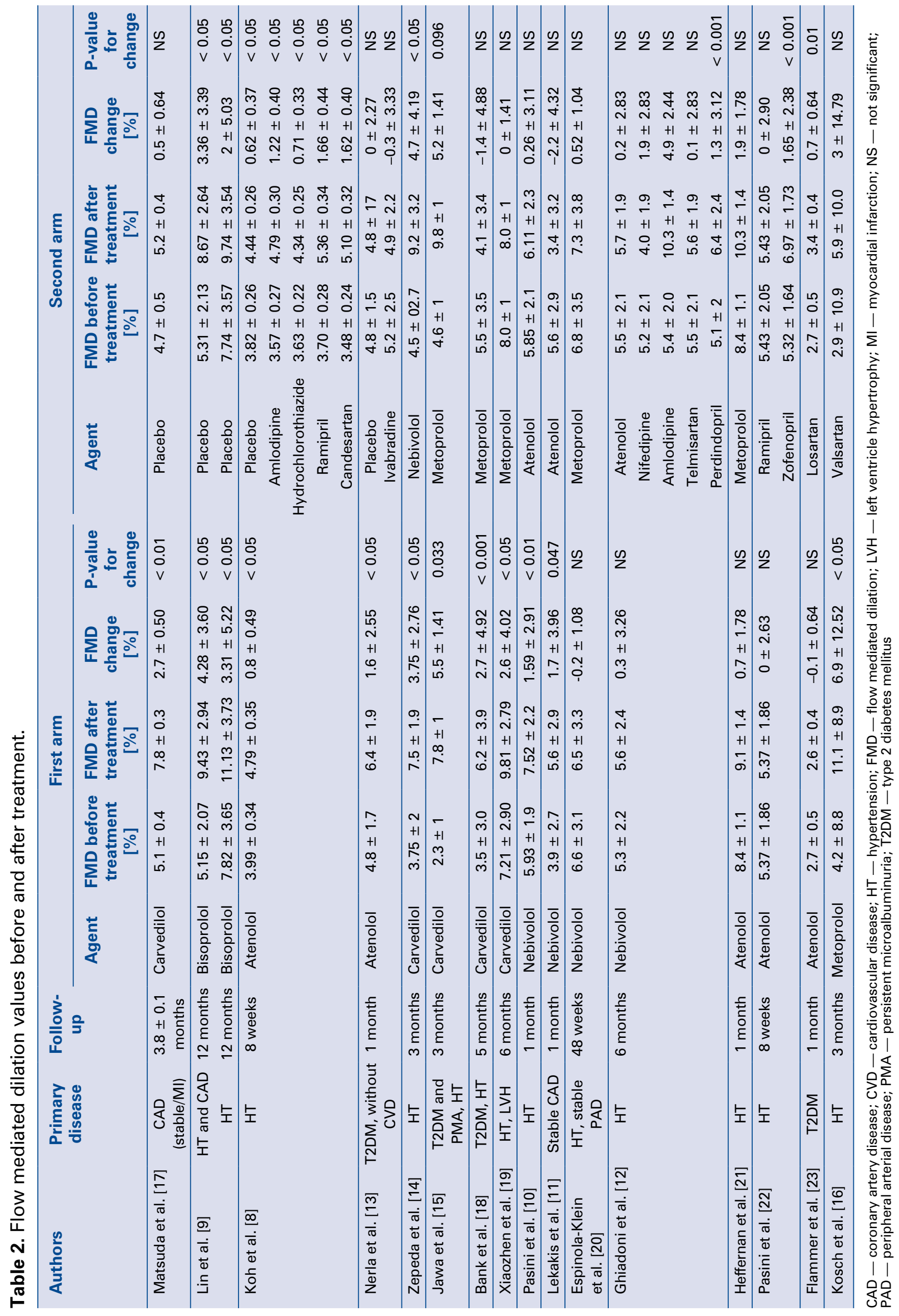




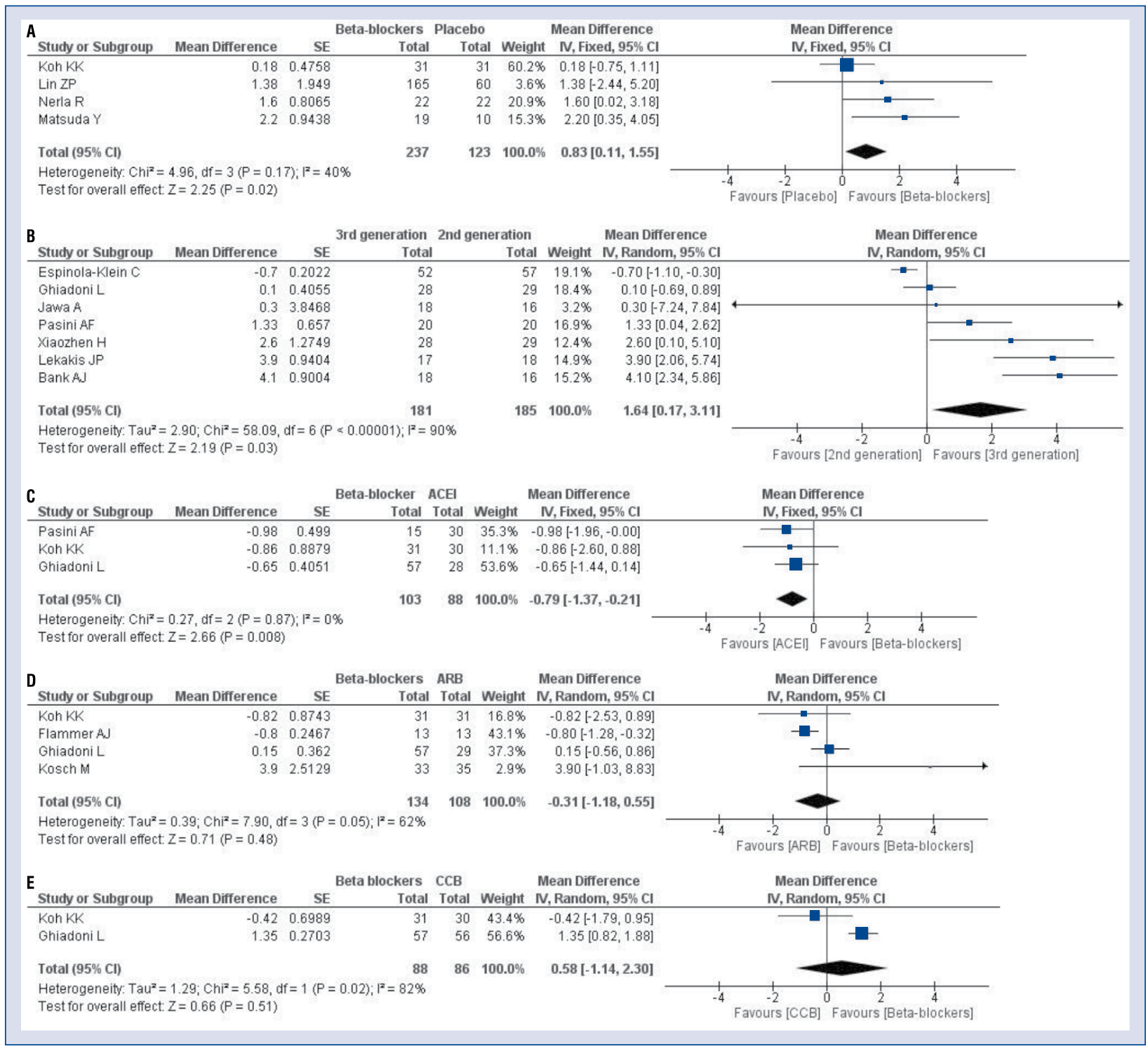

Figure 2. Forest plot presenting effect of (A) beta-blockers and placebo; B. Second and third generation beta-blockers; C. Beta-blockers and angiotensin converting enzyme inhibitors (ACEI); D. Beta-blockers and angiotensin receptor blockers (ARB); E. Beta-blockers and calcium channel blockers (CCB) on flow mediated dilation changes.

assessed as non important, $\mathrm{I}^{2}=40 \% ; \mathrm{p}=0.17$. A forest plot illustrating the comparison between beta-blockers and placebo is presented in Figure 2A.

Third vs. second generation of beta-blockers

Seven of 16 studies, comprising a total of 366 patients compared the effect of $3^{\text {rd }} v s 2^{\text {nd }}$ generation beta-blockers on FMD change [10-12, 15, 18-20]. Period between baseline and final FMD measurements ranged from 1 month to 48 weeks. The $3^{\text {rd }}$ generation beta-blockers investigated were: carvedilol and nebivolol, and the $2^{\text {nd }}$ generation ones: atenolol and metoprolol. The $3^{\text {rd }}$ generation beta-blockers gave a significantly better improvement of FMD compared with $2^{\text {nd }}$ generation beta-blockers, with a MD of $1.65 ; 95 \%$ CI $0.17-3.11 ; \mathrm{p}=0.03$. The heterogeneity of the analyzed trials was assessed as substantial to considerable, $\mathrm{I}^{2}=90 \%$, $\mathrm{p}<0.0001$. A forest plot illustrating the comparison between $3^{\text {rd }}$ and $2^{\text {nd }}$ generation of beta-blockers is shown in Figure 2B. In addition to a $3^{\text {rd }}$ and $2^{\text {nd }}$ generation comparison, Zepeda et al. [14] also compared with each other the effects of two $3^{\text {rd }}$ generation beta-blockers: carvedilol and nebivolol. The two agents gave a similar improvement of FMD, with a MD of: $3.75 \pm 8.67$ for carvedilol and $4.7 \pm$ 
\pm 12.1 for nebivolol ( $p>0.05$ ). Similarly, there was no difference between the $2^{\text {nd }}$ generation beta-blockers: atenolol and metoprolol in the study of Heffernan et al. [21], the mean FMD changes were: $0.70 \pm$ \pm 0.89 and $1.90 \pm 0.89$, respectively $(\mathrm{p}>0.05)$.

\section{Beta-blockers vs. other antihypertensive drugs}

Five of the 16 studies analyzed effects of different classes of antihypertensive drugs on endothelial function $[8,12,16,22,23]$. Three of them involving 191 patients compared the effect of angiotensin converting enzyme inhibitors (ACEI) and beta-blockers on FMD changes $[7,12,23]$. Period between baseline and final FMD measurements varied from 8 weeks to 6 months. The efficacy of beta-blockers was significantly lower compared with ACEI, with the MD of $-0.79 ; 95 \% \mathrm{CI}-1.37-(-0.21) ; \mathrm{p}=0.008$ (Fig. $2 \mathrm{C}$ ). A similar difference was observed when only $3^{\text {rd }}$ generation beta-blockers were compared with ACEI, giving the MD of: -0.97 ; $95 \%$ CI -1.60 $(-0.35) ; \mathrm{p}=0.002$. Four studies including 242 patients with period between FMD measurements ranging from 1 to 6 months compared beta-blockers and angiotensin receptor blockers (ARB) $[8,12$, $16,23]$. The two groups of drugs produced similar FMD changes, with the MD of $-0.31 ; 95 \% \mathrm{CI}$ $-1.18-(-0.55) ; \mathrm{p}=0.48$ (Fig. 2D). The effects of beta-blockers and calcium channel blockers (CCB) on endothelial function were analyzed in two studies including 174 patients with the period between FMD measurements of 8 weeks and 6 months, respectively $[7,12]$. There were no statistically significant differences between the two groups of drugs, the MD being 0.58; 95\% CI -1.14-2.30; $\mathrm{p}=0.51$ (Fig. 2E). Koh et al. [8] compared the effects of beta-blockers and diuretic therapy on FMD change. Beta-blockers and hydrochlorothiazide had similar effects on FMD change: $0.8 \pm 2.04$ and 0.71 \pm 1.94 , respectively $(\mathrm{p}>0.05)$.

\section{Beta-blockers vs. ivabradine}

Nerla et al. [13] compared the effect of atenolol and ivabradine on endothelial function in 41 patients with diabetes mellitus type 2 . After 4 weeks of treatment, the change in the FMD value was significantly higher in the atenolol group than in the ivabradine group: $1.6 \pm 3.61$ vs. $-0.3 \pm 1.66$; $\mathrm{p}=0.02$.

\section{Discussion}

To the best of our knowledge, this is the first meta-analysis of the effects of beta-blockers on endothelial function as assessed by FMD. The main finding of this study is that beta-blockers significantly improve the endothelial function compared with placebo. Moreover, the FMD improvement is significantly higher for $3^{\text {rd }}$ generation beta-blockers compared to the $2^{\text {nd }}$ generation ones. Compared to other antihypertensive drugs, beta-blockers show similar effect on endothelial function as ARB, CCB or diuretics. However, beta-blockers are less effective than ACEI.

The effects of beta-blockers on endothelial function seem to be indirect. There is no strong evidence of an interaction between beta-blockers or their metabolites with endothelial cells. Earlier studies have amply described an impaired endothelial-dependent response of arteries in hypertensive subjects [24]. The pathomechanism of endothelial dysfunction as a response to high blood pressure is intricate. First, higher blood pressure favors reduction of nitric oxide (NO) level, which is predominantly responsible for decreased endothelium-dependent vasodilatation. Persistently decreased shear stress leads to reduction of endothelial nitric oxide synthase (eNOS) level in hypertensive patients [25]. Moreover, hypertension predisposes to higher serum levels of eNOS inhibitors (e.g. asymmetric dimethylarginine) and therefore, additionally results in decreased local NO levels. Second, an increased oxidative stress, due to higher blood pressure is a factor strongly impairing endothelial function [25, 26]. Finally, the damage of endothelial cells caused by high blood pressure is tightly connected with an increased local inflammatory response resulting in remodeling of vessel walls leading to their impaired relaxation [25].

Among the four studies comparing beta-blockers with placebo included in our meta-analysis, only Matsuda et al. [17] studied the effect of $3^{\text {rd }}$ generation beta-blocker (carvedilol). In that study, the improvement of FMD values was the strongest. Carvedilol is a non-selective agent blocking not only beta-adrenergic but also alpha1-adrenergic receptors. Alfa-adrenergic blocking plays a substantial beneficial role in endothelial function improvement [27]. Moreover, the exceptional antioxidant properties of carvedilol may additionally enhance the favorable effect of alpha1-adrenergic receptors blockade on endothelial function [28].

Interestingly, in 2 of the 4 placebo-controlled studies analyzed here, the FMD values remarkably increased also in the placebo groups [8,9]. This situation suggests influence of other factors (e.g., better medical care) on endothelial function despite a lack of hypertension treatment and implies that 
trials with no-treatment group give unrealistically positive outcomes.

An advantage of $3^{\text {rd }}$ generation beta-blockers compared with $2^{\text {nd }}$ generation ones has been shown in this meta-analysis. The beneficial effects of carvedilol on endothelial function improvement are discussed above.

Similarly to beta-blockers, the effect of other antihypertensive drugs on endothelial function seems to be strictly connected with blood pressure reduction. Additionally, the antioxidative properties of ACEI, ARB and CCB are well proven [29-31]. Earlier studies have suggested that the contribution of these agents to oxidative stress reduction is one of the main mechanisms of endothelial function improvement. Some investigators have suggested an important role of antihypertensive drugs in the reduction of inflammation response. However, Koh et al. [8] showed that none of those agents could reduce the $\mathrm{C}$-reactive protein level reduction after 8 weeks of treatment. Noticeably, Nishizaka et al. [32] showed that endothelial function measured with FMD is negatively correlated with serum aldosterone level. The complex effects of ACEI on the renin-angiotensin-aldosterone system can explain the advantage of ACEI over beta-blockers in endothelial function improvement [33].

\section{Limitations of the study}

One of the main limitations of the present meta-analysis is indiscriminate inclusion of studies using both $2^{\text {nd }}$ and $3^{\text {rd }}$ generation beta-blockers. Selection of a single generation of beta-blockers could provide more homogenous subgroups, however, differences within these subgroups can also be significant. Moreover, to increase the number of participants we decided not to limit the analysis to patients with a diagnosed hypertension, in whom the effect of beta-blockers on endothelial function is well studied. The differences between treatment periods in individual studies additionally increased the heterogeneity of data. The strict inclusion criteria according to study the design reduced the number of analyzed trials, but on the other hand contributed to bias reduction. Finally, the lack of exact $p$-values for FMD changes in some studies increased standard error for the differences and reduced the effect of those studies on the metaanalysis results.

\section{Conclusions}

The meta-analysis presented here indicates that beta-blockers improve the endothelial function compared with placebo. Moreover, $3^{\text {rd }}$ generation beta-blockers are significantly better than $2^{\text {nd }}$ generation ones in improving FMD. Beta-blockers have a similar effect on endothelial function as $\mathrm{ARB}, \mathrm{CCB}$ or diuretics, but are inferior in this respect to ACEI.

\section{Conflict of interest: None declared}

\section{References}

1. Halcox JP, Schenke WH, Zalos G et al. Prognostic value of coronary vascular endothelial dysfunction. Circulation, 2002; 106: 653-658.

2. Task Force Members, ESC Committee for Practice Guidelines. 2013 ESC guidelines on the management of stable coronary artery disease: The Task Force on the management of stable coronary artery disease of the European Society of Cardiology. Eur Heart J, 2013; 34: 2949-3003.

3. McMurray JJ, Adamopoulos S, Anker SD et al. ESC Guidelines for the diagnosis and treatment of acute and chronic heart failure 2012. Eur Heart J, 2012; 33: 1787-1847.

4. Ritter JM. Nebivolol: Endothelium-mediated vasodilating effect. J Cardiovasc Pharmacol, 2011; 38: (suppl. 3): S13-S16.

5. Anderson TJ, Meredith IT, Yeung AC, Frei B, Selwyn AP, Ganz P. The effect of cholesterol lowering and antioxidant therapy on endothelium-dependent coronary vasomotion. N Engl J Med, 1995; 332: 488-493.

6. National Collaborating Centre for Methods and Tools (2008). Quality Assessment Tool for Quantitative Studies. Hamilton, ON: McMaster University. (Updated 13 April, 2010).

7. Higgins JPT, Green S eds. Cochrane Handbook for Systematic Reviews of Interventions Version 5.1.0 [updated March 2011]. The Cochrane Collaboration, 2011. Available from www. cochrane-handbook.org.

8. Koh KK, Quon MJ, Han SH et al. Distinct vascular and metabolic effects of different classes of anti-hypertensive drugs. Int J Cardiol, 2010; 140: 73-81.

9. Lin ZP, Dong M, Liu J. Bisoprolol improved endothelial function and myocardium survival of hypertension with stable angina: A randomized double-blinded trial. Eur Rev Med Pharmacol Sci, 2013; 17: 794-801.

10. Pasini AF, Garbin U, Stranieri C. Nebivolol treatment reduces serum levels of asymmetric dimethylarginine and improves endothelial dysfunction in essential hypertensive patients. Am J Hypertens, 2008; 21: 1251-1257.

11. Lekakis JP, Protogerou A, Papamichael C et al. Effect of nebivolol and atenolol on brachial artery flow-mediated vasodilation in patients with coronary artery disease. Cardiovasc Drugs Ther, 2005; 19: 277-281.

12. Ghiadoni L, Magagna A, Versari D et al. Different Effect of Antihypertensive Drugs on Conduit Artery Endothelial Function. Hypertension, 2007; 41: 1281-1286.

13. Nerla R, Di Franco A, Pitocco D et al. Differential effects of heart rate reduction by atenolol or ivabradine on peripheral endothelial function in type 2 diabetic patients. Heart, 2012; 98: 1812-1816.

14. Zepeda RJ, Castillo R, Rodrigo R et al. Effect of carvedilol and nebivolol on oxidative stress-related parameters and endothelial function in patients with essential hypertension. Basic Clin Pharmacol Toxicol, 2012; 111: 309-316. 
15. Jawa A, Nachimuthu S, Pendergrass M, Asnani S, Fonseca V. Beta-blockers have a beneficial effect upon endothelial function and microalbuminuria in African-American subjects with diabetes and hypertension. J Diabetes Complications, 2008; 22: 303-308.

16. Kosch M, Levers A, Lang D, et al. A randomized, double-blind study of valsartan versus metoprolol on arterial distensibility and endothelial function in essential hypertension. Nephrol Dial Transplant, 2008 23: 2280-2285.

17. Matsuda Y, Akita H, Terashima M, Shiga N, Kanazawa K, Yokoyama M. Carvedilol improves endothelium-dependent dilatation in patients with coronary artery disease. Am Heart J, 2000; 140: 753-759.

18. Bank AJ, Kelly AS, Thelen AM, Kaiser DR, Gonzalez-Campoy JM. Effects of carvedilol versus metoprolol on endothelial function and oxidative stress in patients with type 2 diabetes mellitus. Am J Hypertens, 2007; 20: 777-783.

19. Xiaozhen H, Yun Z, Mei Z. Effect of carvedilol on coronary flow reserve in patients with hypertensive left-ventricular hypertrophy. Blood Pressure, 2010; 19: 40-47.

20. Espinola-Klein C, Weisser G, Jagodzinski A et al. Beta-blockers in patients with intermittent claudication and arterial hypertension. Results From the Nebivolol or Metoprolol in Arterial Occlusive Disease Trial. Hypertension, 2007; 58: 148-154.

21. Heffernan KS, Suryadevara R, Patvardhan EA, Mooney P, Karas RH, Kuvin JT. Effect of Atenolol vs Metoprolol Succinate on Vascular Function in Patients With Hypertension. Clin Cardiol, 2011; 34: 39-44.

22. Pasini AF, Garbin U, Nava MC et al. Effect of sulfhydryl and nonsulfhydryl angiotensin-converting enzyme inhibitors on endothelial function in essential hypertensive patients. Am J Hypertens, 2007; 20: 443-450.

23. Flammer AJ, Hermann F, Wiesli P et al. Effect of losartan, compared with atenolol, on endothelial function and oxidative stress in patients with type 2 diabetes and hypertension. J Hypertens, 2007; 25: 785-791.

24. Ward NC, Croft KD, Hodgson J, Rich L, Beilin LJ, Puddey IB. Brachial artery vasomotor function is inversely associated with 24-h ambulatory blood pressure. J Hypertens, 2004; 22: 967-972.

25. Thuillez C, Richard V. Targeting endothelial dysfunction in hypertensive subjects. J Hum Hypertens, 2005; 19 (suppl. 1): S21-25.

26. Klima $€$, Kawecka-Jaszcz K, Stolarz-Skrzypek K et al. Structure and function of large arteries in hypertension in relation to oxidative stress markers. Kardiol Pol, 2013; 71: 917-923.

27. Raij L, Hayakawa H, Coffee K, Guerra J. Effect of doxazosin on endothelial dysfunction in hypercholesterolemic/antioxidantdeficient rats. Am J Hypertens, 2007; 10: 1257-1262.

28. Feuerstein GZ, Ruffolo RR Jr. Carvedilol, a novel vasodilating beta-blocker with the potential for cardiovascular organ protection. Eur Heart J, 1996; 17 (suppl. B): 24-29.

29. Chin BS, Langford NJ, Nuttall SL, Gibbs CR, Blann AD, Lip GY. Anti-oxidative properties of beta-blockers and angiotensinconverting enzyme inhibitors in congestive heart failure. Eur J Heart Fail, 2003; 5: 171-174.

30. Yu Y, Fukuda N, Yao EH et al. Effects of an ARB on endothelial progenitor cell function and cardiovascular oxidation in hypertension. Am J Hypertens, 2008; 21: 72-77.

31. Godfraind T. Antioxidant effects and the therapeutic mode of action of calcium channel blockers in hypertension and atherosclerosis. Philos Trans R Soc Lond B Biol Sci, 2005; 360: 2259-2272.

32. Nishizaka MK, Zaman MA, Green SA, Renfroe KY, Calhoun DA. Impaired endothelium-dependent flow-mediated vasodilation in hypertensive subjects with hyperaldosteronism. Circulation, 2004; 109: 2857-2861.

33. Ferrari R. RAAS inhibition and mortality in hypertension: from pharmacology to clinical evidence. Kardiol Pol, 2013; 71: 1-7. 